\title{
ISOLAMENTO E IDENTIFICAÇÃO DE FUNGOS MICORRÍZICOS RIZOCTONIÓIDES ASSOCIADOS A TRÊS ESPÉCIES DE ORQUÍDEAS EPÍFITAS NEOTROPICAIS NO BRASIL ${ }^{(1)}$
}

\author{
Olinto Liparini Pereira(2), Maria Catarina Megumi Kasuya ${ }^{(3)}$, \\ Christtianno de Lima Rollemberg ${ }^{(4)} \&$ Guilherme Montandon Chaer ${ }^{(5)}$
}

\begin{abstract}
RESUMO
Distúrbios causados pelo homem têm resultado no aumento do risco de extinção de diversos táxons de orquídeas nativas da Mata Atlântica no Brasil. Na natureza, orquídeas utilizam obrigatoriamente fungos endomi corrízicos para a germi nação de sementes e desenvolvimento da plântula, ao menos nos primeiros estádios do seu ciclo de vida. Assim, fungos micorrízicos associados ao sistema radicular de orquídeas nativas vêm sendo isolados, caracterizados e armazenados para uso em futuros programas de conservação de espécies de orquídeas, por meio da germinação simbiótica. Três isolados de fungos micorrízicos rizoctonióides foram obtidos do sistema radicular de três espécies de orquídeas neotropicais, Gomesa crispa, Campyl ocentrum organensee Bul bophyl lum sp., de três diferentes fragmentos de Mata Atlântica no Brasil. Estudos taxonômicos, baseados na condição nuclear, morfologia da hifa vegetativa e ultra-estrutura do septo doli pórico, revelaram que os isolados pertencem aos gêneros Ceratorhiza e Rhizoctonia. Esse é o primeiro relato do isolamento de fungos micorrízicos associados ao sistema radicular dessas espécies de orquídeas neotropicais. Aspectos relativos à taxonomia e ao uso desses isolados no contexto de um programa de conservação de orquídeas nativas são discutidos.
\end{abstract}

Termos de indexação: biodiversidade, ecologia microbiana, micologia, endomicorrizas, Ceratorhiza, Rhizoctonia.

\footnotetext{
(1) Trabalho desenvol vido no Laboratório de Associações Micorrízicas, Instituto de Biotecnol ogia Aplicada à Agropecuária, BI OAGRO, Departamento de Microbiologia, U niversidade Federal de Viçosa - UFV. Recebido para publicação em março de 2004 e aprovado em dezembro de 2004

(2) Mestre em Microbiologia Agrícola, BIOAGRO, Universidade Federal deViçosa - UFV. Departamento de Microbiologia, CEP 36570000 Viçosa (MG). Bolsista CNPq. E-mail: liparini@bol.com.br

(3) Professora Adjunta do Departamento de Microbiologia, UFV. Bolsista CNPq. E-mail: mkasuya@ufv.br

(4) Bolsista de Iniciação Científica PIBIC/CNPq. E-mail: christthroll@yahoo.com

(5) Pesquisador da E mbrapa-Agrobiologia. Caixa Postal 74505, CEP 23851-970 Seropédica (RJ ). E-mail: gchaer@cnpab.embrapa.br
} 


\title{
SUMMARY: ISOLATION AND IDENTIFICATION OF RHIZOCTONIA-LIKE MYCORRHIZAL FUNGI ASSOCIATED TO THREE NEOTROPICAL EPIPHYTIC ORCHID SPECIES IN BRAZIL
}

\begin{abstract}
Anthropogenic disturbances haveresulted in an increased threat of extinction of many native orchid taxa in Brazil's Atlantic rain forest. In nature, orchids utilize mycorrhizal fungi to initiate seed germi nation and seedling devel opment, at least in the early stages of their lifecycle. Mycorrhizal fungi associated with theroots of orchids havethus been isolated, characterized and stored as i mportant resources for a future conservation program of orchid species through symbi otic seed germi nation. Threemycorrhizal Rhizoctonia-likefungi were isolated from roots of three neotropical orchid species Gomesa crispa, Campylocentrum organense and Bulbophyllum sp. from three different Atlantic rain forest fragments in Brazil. Taxonomic studies based on the nuclear condition, vegetative hyphal morphology and septal pore ul trastructure revealed that the isolates bel ong to the genera Ceratorhiza and Rhizoctonia. This is the first report on the isolation of mycorrhizal fungal species associated to the referred neotropical orchid species. Aspects concerning their taxonomy and use in the context of a native orchid conservation programs are discussed.
\end{abstract}

Index terms: biodiversity, microbial ecology, mycology, endomycorrhizae, Ceratorhiza, Rhizoctonia.

\section{NTRODUÇÃO}

A família Orchidaceae é uma das mai ores e mais diversificadas do reino vegetal, com várias espécies registradas no mundo inteiro, dentre terrestres, epífitas e rupícolas (Cronquist, 1981; Dressler, 1993). O Brasil dispõe de grande biodiversidade dessas espécies, principalmente das epífitas. No entanto, o desbravamento de áreas, como as do cerrado, e a derrubada de matas, como a mata atlântica e a floresta amazônica, têm ameaçado e levado à extinção algumas dessas espécies (Rushi, 1986; Miller \& Warren, 1996; Costa et al., 1998). Muitas espécies, raramente observadas na natureza, encontram-se em diversas listas de prioridade máxima de conservação, por apresentarem baixo sucesso reprodutivo ou habitat restrito (Mendonça \& Lins, 2000) e mesmo espécies ainda não listadas encontram-seameaçadas, justificando trabal hos que visem à sua conservação e reintrodução em habitat nativo. Entretanto, a organização eficiente de programas de reintrodução, conservação e manejo de orquídeas na natureza requer um conhecimento detalhado das estratégias reprodutivas dessas espécies em seu habitat natural (Clements et al., 1986; Pereira et al., 2003b).

Na natureza, as orquídeas necessitam seassociar, obrigatoriamente, a fungos micorrízicos para a germinação da semente e nutrição, sendo esses de fundamental importância para que a espécie possa completar o seu ciclo de vida (Leake, 1994; Smith \& Read, 1997; Peterson et al., 1998; Rasmussen, 2002; Pereira et al., 2003b). Especula-se, também, o importante papel de determinadas bactérias associadas ao sistema radicular de orquídeas
(Wilkinson et al., 1994; Rasmussen, 2002), havendo, inclusive, relato da ocorrência de fixadores de $\mathrm{N}_{2}$ no substrato de crescimento e na ecto e endorrizosfera de várias espécies de orquídeas coletadas no Brasil (Lange \& Moreira, 2002).

Diante do exposto, o conhecimento dos fungos micorrízicos associados ao sistema radicular, bem como o seu uso em programas de propagação simbiótica de orquídeas, é de grande importância para a reintrodução, conservação e manejo dessas espécies vegetais (Zettler, 1997a,b; Pereira et al., 2003b).

Os trabalhos sobre mi corrizas em orquídeas têmse concentrado em espécies terrestres de ambientes temperados e poucos trabal hos com espécies epífitas ou de ambiente tropical existem na literatura (Peterson et al., 1998; Pereira et al., 2003a; Kasuya et al., 2003).

O objetivo destetrabal ho foi verificar a ocorrência de fungos micorrízicos em três espécies de orquídeas neotropicais, identificando-os e isolando-os em cultura pura para depósito no banco degermoplasma de fungos micorrízicos de orquídeas do Laboratório de Associações Micorrízicas/BIOAGRO/UFV, com vistas em futura utilização em programas de germinação simbiótica de sementes.

\section{MATERIAL E MÉTODOS}

Raízes saudáveis de Campyl ocentrum organensis (RchB. f.) Rolfe, Bulbophyllum sp. e Gomesa crispa (Lindl.) Klotzsch ex RchB. f. foram amostradas de plantas selvagens em ambiente natural. As espécies 
foram sel ecionadas, considerando a el evada concentração de plântulas em germinação próximo ao seu sistema radicular, indicando a possível presença de fungos micorrízicos em níveis elevados. Dessas amostras, partefoi seccionada transversalmenteem criomicrótomo (LEICA CM 1850), na espessura de $30 \mu \mathrm{m}$, para exame em microscópio ótico, quanto à presença de pelotons nas células corticais, e para registro fotográfico. Os fungos micorrízicos foram isolados diretamente de pelotons não degradados (Pereira, 2001), assegurando, assim, o isolamento somente dos fungos formadores dessas estruturas. Todo mi cél io característico de fungo rizoctonióide foi transferido para meios de cultura e subcultivados repetidamente até à obtenção de culturas puras (Sneh et al., 1991). Somente isolados reconhecidamente pertencentes ao grupo defungos rizoctonióides, capazes de formar células monilióides (Roberts, 1999), foram selecionados para a identificação. A indução da formação de células monilióides foi feita em mei o fubá (Pereira, 2001), assim como a indução de fase sexuada (Currah, 1986). Para indução da fase sexuada, os fungos cultivados em placas que continham mei o fubá foram incubados no escuro por $48 \mathrm{~h}$ a $25^{\circ} \mathrm{C}$ e, posteriormente, submetidos à alternância de $12 \mathrm{~h}$ de luz próximo ao ultraviol eta / $12 \mathrm{~h}$ escuro (Leach, 1962) a $25^{\circ} \mathrm{C}$.

A identificação dos isolados foi feita em cultura, com base na condição nuclear e ultra-estrutura do septo dolipórico (Moore, 1987; Andersen, 1996; Andersen \& Rasmussen, 1996). Para a col oração de núcleos, microculturas dos isolados foram preparadas entrelâmina elamínula (Hawksworth, 1974) e, após incubação por 7 d a $25^{\circ} \mathrm{C}$, as lamínulas foram montadas com $50 \mu \mathrm{L}$ de uma sol ução corante que continha $1 \mu \mathrm{L} \mathrm{L}^{-1} 10.000 \times \mathrm{SYBR}{ }^{\circledR}$ Green I em $\mathrm{KH}_{2} \mathrm{PO}_{4} 10 \mathrm{mmol} \mathrm{L}^{-1}$ e glicerol a $18 \%$ (Meinhardt et al., 2001). Após 5 min em câmara escura, as lâminas foram observadas em mi croscópio ótico Nikon E600 acoplado de epifluorescência Y-F L operando na faixa decomprimento deonda de 450-520 nm deexcitação.

Para a observação da ultra-estrutura do septo dolipórico, discos com mi célio dos isolados fúngicos, cultivados em meio batata-dextrose-ágar (BDA) a $28{ }^{\circ} \mathrm{C}$, por sete dias, foram fixados em solução de glutaraldeído $3 \%$ preparado em tampão cacodilato $0,05 \mathrm{~mol} \mathrm{~L}^{-1}, \mathrm{pH} 7,2$, durante $2 \mathrm{~h}$, à temperatura ambiente. Após a fixação, o material foi submetido a seis lavagens de $10 \mathrm{~min}$ cada com tampão cacodilato. Foi realizada uma pós-fixação com solução de tetraóxido de ósmio $1 \%$ no mesmo tampão, por $2 \mathrm{~h}$, à temperatura ambiente. A seguir, o material foi lavado em tampão cacodilato e desidratado em série al coólica que continha concentrações de 30, 50, 70, 80 e 95 \% deál cool, pela imersão, durante 10 min, em cada uma das sol uções, e mais duas vezes em ál cool absoluto, por 20 min. O material foi embebido em resina Spurr adicionando resina às amostras. As amostras foram imersas em ál cool absoluto, na proporção de ál cool:resina de 1:1 e 1:3 com intervalo de 30 min entre cada troca e, a seguir, em resina pura, por $5 \mathrm{~h}$, na primeira troca, e, para $12 \mathrm{~h}$, na segunda troca. O material foi montado em formas, enquanto a resina foi polimerizada em estufa a $70^{\circ} \mathrm{C}$ por $24 \mathrm{~h}$.

A obtenção de cortes semifinos foi realizada em ultrami crótomo Sorvall, utilizando naval ha de vidro sendo os cortes tratados com azul de toluidina para localização de regiões do micélio do fungo que possibilitavam a visualização dos septos. Os cortes ultrafinos foram feitos com o mesmo equipamento, sendo eles montados em grades de Cu previamente recobertas com Formvar preparadoem sol ução 0,3 \% em clorofórmio. A contrastação do material foi efetuada pela imersão em solução de acetato de uranila, por $30 \mathrm{~min}$, seguida de citrato de $\mathrm{Pb}$, por 15 min. Finalmente, os cortes foram levados à observação em mi croscópio el etrôni co detransmissão (Zeiss EM 109), operando a 80 kV, e as regiões onde era possível a distinção do septo dolipórico foram fotografadas, utilizando-se filme de $35 \mathrm{~mm}$.

\section{RESULTADOS E DISCUSSÃO}

Foram observados pel otons nos cortes transversais das raízes de G. crispa, C. organense e Bulbophyllum sp., apresentando o sistema radicular de G. crispa mais de $90 \%$ das células do córtex colonizadas (Figura $1 \mathrm{~A}$ ), enquanto as demais espécies apresentavam cerca de $50 \%$ de colonização (F igura 1B), como observado em outras espécies de orquídeas epífitas tropicais (Pereira, 2001). Os pel otons apresentavam-se bem distribuídos por todo o córtex da raiz e em todos os estádios de desenvolvimento, aparentemente intactos ou degradados (Figura 1C). Em G. crispa e Campyl ocentrum sp., foi observada a presença de hifas fora da região do córtex da raiz, na extensão da superfície externa do velame. Essetipo de distribuição não écomum, mas tem si do observado em fungos rizoctoniói des encontrados associados às espécies de orquídeas epífitas tropicais (Richardson et al., 1993; Pereira, 2001).

O padrão de colonização mais comumente observado nos cortes das raízes amostradas consistia na presença de pelotons não degradados, circundando toda a região mais externa do córtex, próximo à superfície interna do velame e de novel os dehifas digeridas na região mediana e mais interna do córtex (Figura 1C). A presença de pelotons intactos circundando a região mais externa do córtex da raiz talvez sirva como uma importante fonte de inóculo para a manutenção de hifas na superfície externa do vel ame deorquídeas epífitas, promovendo a infecção de sementes depositadas junto ao sistema radicular de plantas adultas na natureza (Pereira et al., 2005). O padrão de colonização com sincronismo de pelotons intactos e degradados em toda região do córtex, como comumente observado 
em orquídeas terrestres de clima temperado (Zelmer et al., 1996), não foi constatado. Os fungos isolados diretamente dos pel otons eram todos rizoctonióides, pertencentes aos gêneros: Rhizoctonia ou Ceratorhiza (Quadro 1).

Os três isolados obtidos de pelotons intactos mostravam ramificação de hifas em ângulo reto e constrição da hifa na altura do septo (Figura 1D e E), características marcantes do micélio vegetativo de rizoctonias (Sneh et al., 1991). Em BDA, os isolados M8 e M10 apresentavam micélio aéreo abundante, inicialmente creme, tornando-se amarronzado em poucos dias, enquanto o isol ado M 9 mostrava micélio branco. Todos os isolados apresentavam micélio vegetativo consistindo de hifas septadas, sem grampo de conexão, diâmetro superior a 4,0 $\mu \mathrm{m}$, paredelisa epouco espessa. Todos os isolados formaram células monilióides em meio fubá (Figura 1D), após quatro semanas de incubação, as quais se apresentavam cilíndricas ou na forma de barril esem espessamento de parede. A formação de células monilióides caracteriza os fungos pertencentes ao grupo dos fungos rizoctonióides (Roberts, 1999). O isolado M 10 destaca-se pela produção de células monilióides em grande quantidade, arranjadas à semelhança de microescleródios (Figura IF) e de coloração róseoalaranjada quando expostas à iluminação. Nenhum himênio pertencente à fase sexuada foi observado em mei o fubá, caracterizando a não-indução de fase sexuada pelo método utilizado. O insucesso na indução de fase sexuada em cultura é comumente relatado para diversos isolados de fungos micorrízicos de orquídeas (Zelmer et al., 1996; Pereira, 2001; Shan et al., 2002).

Richardson et al. (1993) relataram o isolamento de Ceratorhiza goodyerae-repentis do sistema radicular de Campylocentrum mi cranthum (Lindl.) Rolfe, enquanto Otero et al. (2002), em estudo filogenético, relataram o isol amento de Ceratorhiza do sistema radicular de Campylocentrum fasciola (Lindl.) Cogn. e Campylocentrum filiforme (Sw.) Cogn. O gênero de fungo rizoctoniói de Ceratorhiza parece estar associado ao gênero Campyl ocentrum. Para o isolado de Ceratorhiza M9 obtido de C. organense neste trabalho, bem como para outros isolados de Ceratorhiza (M2, M3, M4, M7 e M8) previamente isolados de orquídeas epífitas neotropicais (Pereira, 2001), não foi identificada a espécie, uma vez que a taxonomia da espécie para Ceratorhiza e Rhizoctonia, baseada em caracteres morfológicos específicos, é extremamente difícil, pois o fungo raramente apresenta uma característica peculiar da espécie que não mostre variação com o tipo de meio de cultivo utilizado (Andersen, 1990). O trabalho de Richardson et al. (1993) constitui o primeiro relato de Ceratorhiza associado ao gênero Campylocentrum; entretanto, C. goodyeraerepentis é considerado táxon inválido (Andersen, 1990). Portanto, a comparação do isolado M9 com as características morfológicas relatadas para C. goodyeraerepentis não permite afirmar tratar-se da mesma espécie ou de espécies diferentes de Ceratorhiza associadas ao gênero Campyl ocentrum. Para esses casos, o uso de ferramentas moleculares pode auxiliar a elucidar a existência de identidade genética entre diferentes isolados de fungos micorrízicos rizoctonióides (Pereira, 2001; Otero et al., 2002; Sel osse et al., 2002; Shan et al., 2002).

Dentreas espécies conhecidas deCampyl ocentrum, C. organense é considerada altamente vulnerável, com alto risco de extinção (Miller \& Warren, 1996). $\mathrm{O}$ isolado M9 pode ser de grande valia em futuros programas de reintrodução de Campyl ocentrum na natureza, por mei o do cultivo simbiótico desementes. A mesma perspectiva pode ser sugerida em relação ao uso dos isolados M 8 eM 10 em futuros programas de reintrodução de espécies de orquídeas ameaçadas de extinção em seu habitat natural. Em ambos os casos, é fundamental o estudo prévio da compati bilidade dos isol ados em relação às espécies de orquídeas a serem micorrizadas, a exemplo da seleção de isolados de fungos ectomicorrízicos compatíveis, previamente ao estabel ecimento de programas de micorrização controlada em espécies florestais (Costa et al., 2003).

A condição multinucleada do isolado M8 de G. crispa (Figura 1G) asseguraria sua classificação como pertencente ao gênero Moniliopsis (Moore, 1987). Moore propõe o gênero Moniliopsis para acomodar anamorfos com teleomorfos em Thanatephorus e Waitea (ambos com células multinucleadas); entretanto, embora a proposta esteja de acordo com o Código Internacional de Nomenclatura Botânica, o nome Rhizoctonia foi mantido por conservação (nomina conservanda) em

Quadro 1. Código, hospedeiro, condição nuclear, tipo de parenteossoma e gênero dos três isolados de fungos micorrízicos das orquídeas estudadas

\begin{tabular}{cllll}
\hline Código do solado & \multicolumn{1}{c}{ Hospedeiro } & Condição nuclear & Parenteossoma & Gênero \\
\hline M8 & Gomesa crispa & multinucleado & perfurado & Rhizoctonia \\
M9 & Campylocentrum organense & binucleado & perfurado & Ceratorhiza \\
M10 & Bulbophyllum sp. & binucleado & perfurado & Ceratorhiza \\
\hline
\end{tabular}



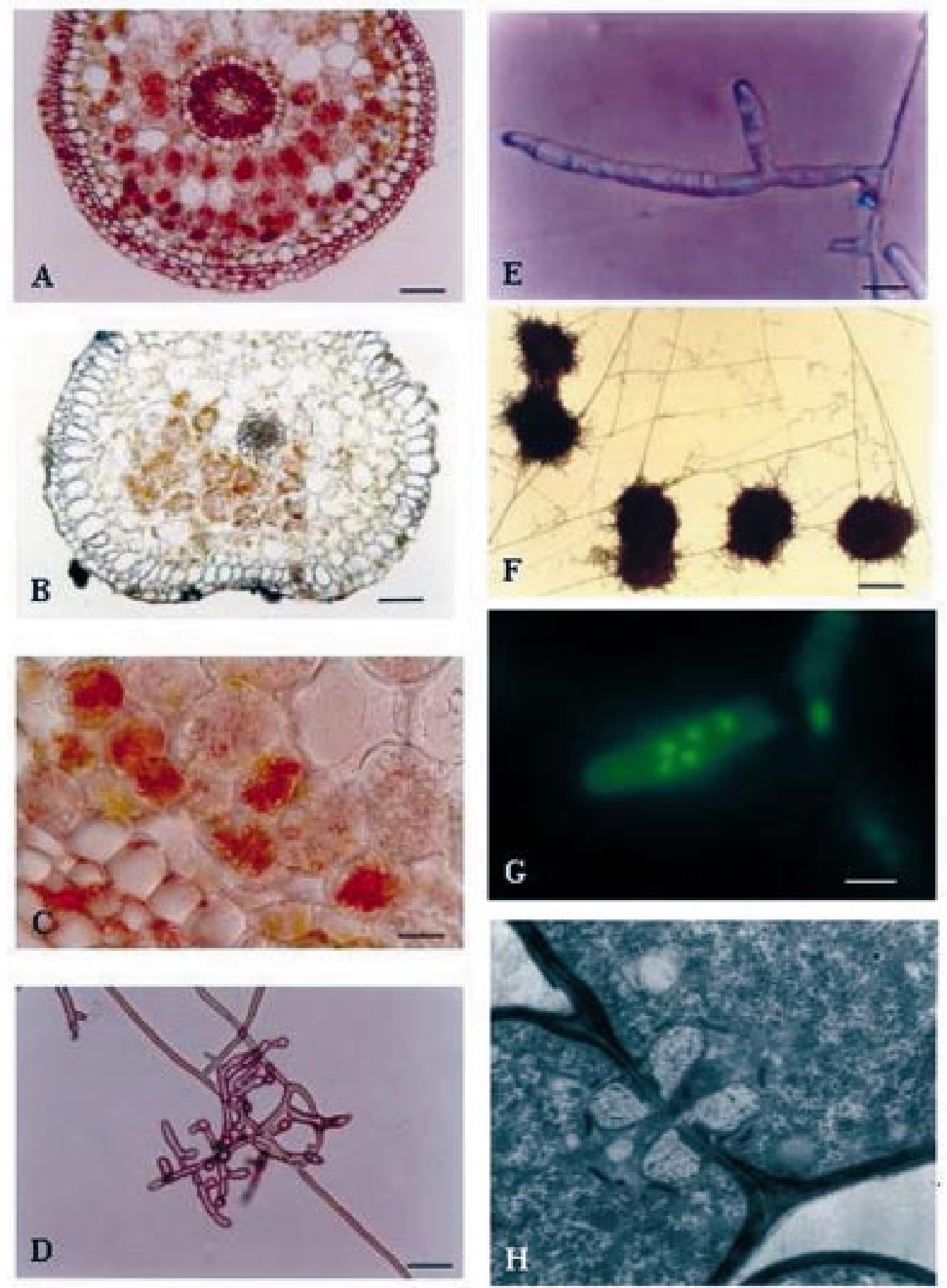

Figura 1. (A) Corte transversal de raiz de Gomesa cri spa e de (B) Campyl ocentrum organense, notando-se a colonização das células do córtex; $(C)$ detalhe da visualização de pelotons intactos, notando-se a presença de hifas amarronzadas, septadas e individualizadas e pelotons digeridos, notando-se a presença de massa fúngica sem hifas individualizadas; (D) Células moniliói des de Rhizoctonia isolada do sistema radicular de G. crispa formadas após incubação por quatro semanas em ágar fubá; (E) Hifa vegetativa gutulada de Ceratorhiza, isolado M9, com ramificação em ângulo reto e constrição da hifa na altura do septo; (F) Microescleródios de Ceratorhiza, isolado M10, formados após incubação por cinco semanas em ágar fubá; (G) Célula multinucleada de Rhi zoctonia, isolado M8, corada com SYBRâ Green I e (H) Microscopia eletrônica de transmissão da secção longitudinal da hifa vegetativa de Ceratorhiza, isolado M9, mostrando a ultra-estrutura do septo dolipórico, as setas indicam o parenteossomo do tipo perfurado. Barras: (A) e (B) $=10 \mu \mathrm{m}$; (C) e (E) $=20 \mu \mathrm{m}$; (D) $=40 \mu \mathrm{m}$; (F) = $100 \mu \mathrm{m} ;(\mathrm{G})=4,0 \mu \mathrm{m}$ e (H) aumento de 25.000X. 
razão da importância econômica desse táxon e do número de trabalhos já publicados, utilizando o nome Rhizoctonia (Stal pers et al., 1998). O gênero Rhizoctonia foi descrito por DeCandole em 1815, sendo R. sol ani a mais conheci da espécie deste gênero por tratar-se de um fungo de ampla distribuição geográfica, de grande importância econômica, fitopatogênico de diversas espécies vegetais (Ogoshi, 1996). Além de reconhecido fitopatógeno, Rhizoctonia é também relatado em associações micorrízicas (Andersen \& Rasmussen, 1996) e endofíticas (Bayman et al., 1997) com orquídeas.

No Brasil, os primeiros estudos taxonômicos de fungos micorrízicos em orquídeas nativas relatam o isol amento de Ceratorhiza spp. a partir de raízes de I sochiluslineares $(\mathrm{V}$ acq.) $\mathrm{R}$. Br., Maxillaria marginata Fenzl., Oncidium flexuosum (Kunth) Lindl. e Oncidium varicosum Lindl. \& Paxton (Pereira, 2001); E pul orhiza repens de Oeceoclades maculata (Lindl.) Lindl. (Pereira et al., 2001), eE pulorhiza epi phytica Pereira, Rollemberg et Kasuya de Epidendrum rigi dum J acq. e Polystachya concreta (J acq.) Garay \& Sweet (Pereira et al., 2003a). Nenhum fungo micorrízico pertencente ao gênero Rhizoctonia foi previamente relatado em orquídea brasileira.

Os três isolados de fungos micorrízicos apresentaram ultra-estrutura do septo como sendo do tipo dolipórico, com parenteossoma do tipo perfurado (descontínuo). Ceratorhiza e Rhizoctonia possuem, respectivamente, tel eomorfos em Ceratobasi dium e Thanatephorus, ambos pertencentes à família Ceratobasidiaceae (Basidiomycetes). À exceção dos Ustilaginales, que apresentam septo simples, todos os outros basi diomi cetos apresentam el aborado aparato do poro do septo, denominado estrutura doliporo/parenteossomo (D/P) (Moore \& McAlear, 1962). Os três isolados dispunham de estrutura D/ $\mathrm{P}$ com quatro largas perfurações (Figura $1 \mathrm{H}$ ), como já observado em Ceratorhiza e R hizoctonia isoladas ou micorrízicas de orquídeas terrestres de ambientetemperado (Andersen, 1996; Currah \& Sherburne, 1992).

Os isolados foram armazenados em discos de BDA contendo micélio fúngico imersos em água destilada esterilizada a $4{ }^{\circ} \mathrm{C}$ em câmara fria (Sneh \& Adams, 1996), como parte da micoteca do Laboratório de Associações Micorrízicas do Departamento de Microbiologia/UFV, com o código de registro (Quadro 1).

\section{CONCLUSÕES}

1. Fungos micorrízi cos rizoctonióides mostraramseassociados aosistema radicular deCampyl ocentrum organense, Bulbophyllum sp. e Gomesa crispa.

2. O fungo associado a Campylocentrum organensee Bul bophyll um sp. foi identificado como Ceratorhiza e à Gomesa crispa como Rhizoctonia.
3. Este foi o primeiro relato de Rhizoctonia micorrízica isolada de orquídea epífita no Brasil.

\section{AGRADECIMENTOS}

Ao Instituto Estadual de Florestas (IEF), pela concessão de registro para coleta de orquídeas e sementes em áreas de conservação biológica do estado de Minas Gerais. Este trabalho é parte integrante do estudo da biodiversidade de fungos micorrízicos associados ao sistema radicular de orquídeas brasileiras financiado pela Fundação de Amparo à Pesquisa do estado de Minas Gerais (FAPEMIG), registro 708/02. Ao CNPq, pela concessão de bolsas de pesquisa; ao Núcleo de Microscopia e Microanálise da UFV; ao Prof. Kiyoshi Matsuoka, pela orientaçãoem microscopia eletrônica de transmissão; à Sociedade Brasileira de Orquidófilos, eao Ph.D. Larry Peterson, University of Guel ph (Canadá), pel o constanteapoio eincentivo.

\section{LITE RATURA CITADA}

ANDERSEN, T.F. \& RASMUSSEN, H.N. The mycorrhizal species of Rhizoctonia. In: SNEH, B.; J ABAJ I-HARE, S.; NEATE, S. \& DIJ ST, G., eds. Rhizoctonia species: taxonomy, molecular biology, ecology, pathology, and disease control. Dordrecht, Kluwer Academic Publishers, 1996. p.379-390.

ANDERSEN, T.F. A comparativetaxonomic study of Rhizoctonia sensu lato employing morphological, ultrastructural and molecular methods. Mycol. Res., 100:1117-1128, 1996.

ANDERSEN, T.F. A study of hyphal morphology in the form genus Rhizoctonia. Mycotaxon, 37:25-46, 1990.

BAYMAN, P.; LEBRÓN, L.; TREMBLAY, R.L. \& LODGE, D.J . Variation in endophytic fungi from roots and leaves of Lepanthes (Orchidaceae). New Phytol., 135:143-149, 1997.

CLEMENTS, M.A.; MUIR, H.\& CRIBB, P.J. A preliminary report on the symbiotic germination of European terrestrial orchids. Kew Bull., 41:437-445, 1986.

COSTA, C.M.R.; HERMANN, G.; MARTINS, C.S.; LINS, L.V. \& LAMAS, I.R. Biodiversidade em Minas Gerais: um atlas para sua conservação. Belo Horizonte, Fundação Biodiversitas, 1998. 94p.

COSTA, M.D.; PEREIRA, O.L.; KASUYA, M.C.M. \& BORGES, A.C. Ectomicorrizas: A face oculta das florestas - aplicações biotecnológicas das ectomicorrizas na produção florestal. Biotec. Ci. Desenvol., 29:38-46, 2003.

CRONQUIST, A. An integrated system of classification of flowering plants. New York, Columbia University Press, 1981. 1262p.

CURRAH, R.S. \& SHERBURNE, R. Septal ultrastructure of some fungal endophytes from boreal orchid mycorrhizas. Mycol. Res., 96:583-587, 1992. 
CURRAH, R.S. Thanatephorus pennatus sp. nov. isolated from mycorrhizal roots of Calypso bulbosa (Orchidaceae) from Alberta. Can. J . Bot., 65:1957-1960, 1986.

DRESSLER, R.L. Phylogeny and classification of the Orchid Family. Cambridge, Cambridge University Press, 1993. 314p.

HAWKSWORTH, D.L. Mycologist's handbook. Kew, Commonwealth Mycological Institute, 1974. 231p.

KASUYA, M.C.M.; PEREIRA, O.L.; ROLLEMBERG, C.L.; BORGES, A.C. \& ARAÚJ O, E.F. Mycorrhizal fungi from neotropical Brazilian orchids. In: INTERNATIONAL CONFERENCE ON MYCORRHIZAE (ICOM), 4. Montreal, 2003. Anais. Montreal, Canadian Society of Soil Science and Canadian Society of Agronomy, 2003. p.414.

LANGE, A. \& MOREIRA, F.M.S. Detecção de Azospirillum amazonense em raízes e rizosfera de Orchidaceae e de outras famílias vegetais. R. Bras. Ci. Sol o, 26:529-533, 2002.

LEACH, C.M. Sporulation of diverse species of fungi under nearultraviolet radiation. Can. J . Bot., 40:151-161, 1962.

LEAKE, J . The biology of myco-heterotrophic ('saprophytic') plants. New Phytol., 127:171-216, 1994.

MEINHARDT, L.W.; BELLATO, C.M. \& TSAI, S.M. SYBR ${ }^{\circledR}$ Green I used to evaluate nuclei number of fungal mycelia. Biotechniques, 31:42-46, 2001.

MENDONÇA, M.P. \& LINS, L.V. Lista vermelha das espécies ameaçadas de extinção da flora de Minas Gerais. Belo Horizonte, Fundação Biodiversitas \& Fundação ZôoBotânica de Belo Horizonte, 2000. 157p.

MILLER, D. \& WARREN, R. Orquídeas do Alto da Serra - da mata atlântica pluvial do sudeste do Brasil. Rio deJ aneiro, Salamandra, 1996. 256p.

MOORE, R.T. \& MCALEAR, J.H. Fine structure of mycota. 7. Observations on septa of ascomycetes and basidiomycetes. Am. J . Bot., 49:86-94, 1962.

MOORE, R.T. The genera of Rhizoctonia-like fungi: Ascorhizoctonia, Ceratorhiza gen. nov., E pulorhiza gen. nov., Moniliopsis, and Rhizoctonia. Mycotaxon, 29:91-99, 1987.

OGOSHI, A. Introduction - The Genus Rhizoctonia. In: SNEH, B.; J ABAJ I-HARE, S.; NEATE, S. \& DIJ ST, G., eds. Rhizoctonia species: taxonomy, molecular biology, ecology, pathology, and disease control. Dordrecht, Kluwer Academic Publishers, 1996. p.1-9.

OTERO, J . T.; ACKERMAN, J . D. \& BAYMAN, P. Diversity and host specificity of endophytic Rhizoctonia-like fungi from tropical orchids. Am. J . Bot., 89:1852-1858, 2002.

PEREIRA, O.L.; KASUYA, M.C.M.; BORGES, A.C.\& ARAÚJ O, E.F. Morphological and molecular characterization of mycorrhizal fungi isolated from neotropical orchids in Brazil. Can. J . Bot., 83:54-65, 2005.

PEREIRA, O.L. Caracterização morfológica e molecular de fungos micorrízicos de sete espécies de orquídeas neotropicais. Viçosa, U niversidade Federal deViçosa, 2001. 48p. (Tese de Mestrado)

PEREIRA, O.L.; ROLLEMBERG, C.L.; BORGES, A.C.; MATSUOKA, K. \& KASUYA, M.C.M. Epulorhiza epiphytica sp. nov. isolated from mycorrhizal roots of epiphytic orchids in Brazil. Mycoscience, 44:153-155, 2003a.
PEREIRA, O.L.; ROLLEMBERG, C.L. \& KASUYA, M.C.M. Association des mycorhizies dans les orchidees perspectives d'utilisation dans les programmes de propagation symbiotique. Orchidees, 55:24-27, 2003b.

PEREIRA, O.L.; ROLLEMBERG, C.L.; PEIXOTO, H.T.M.; ARAÚJ O, E.F.; BORGES, A.C. \& KASUYA, M.C.M. Epulorhiza repens: a orchid mycorrhizal fungus of Oeceodlades maculata. In: CONGRESSO BRASI LEIRO DE MICROBI OLOGIA, 21., Foz do I guaçu, 2001. Anais. F oz do I guaçu, Sociedade Brasileira de Microbiologia, 2001. p.259.

PETERSON, R. L.; UETAKE, Y. \& ZELMER, C. Fungal simbiosis with orchid protocorns. Symbiosis, 25:29-55, 1998.

RASMUSSEN, H.N. Recent developments in the study of orchid mycorrhiza. Plant Soil, 244:149-163, 2002.

RICHARDSON, K.A.; CURRAH, R.S. \& HAMBLETON, S. Basidiomycetous endophytes from the roots of neotropical epiphytic orchidaceae. Lindleyana, 8:127-137, 1993.

ROBERTS, P. Rhizoctonia-forming fungi. Kew, Whitstable Litho Printers, 1999. 239p.

RUSCHI, A. Orquídeas do estado do Espírito Santo. Rio de J aneiro, Expressão e Cultura, 1986. 278p.

SELOSSE, M.A.; WEIâ, M.; JANY, J.L. \& TILLIER, A. Communities and populations of sebacinoid basidiomycetes associated with the achlorophyllous orchid Neottia nidusavis (L.) L.M.C. Rich. and neighbouring tree ectomycorrhizae. Mol. Ecol., 11:1831-1844, 2002.

SHAN, X.C.; LIEW, E.C.Y.; WEATHERHEAD, M.A. \& HODGKISS, I.J. Characterization and taxonomic placement of Rhizoctonia-like endophytes from orchid roots. Mycologia, 94:230-239, 2002.

SMITH, S.E. \& READ, D.J. Mycorrhizal symbiosis 2.ed. San Diego, Academic Press, 1997. 605p.

SNEH, B. \& ADAMS, G.C. Culture preservation methods for maintaining the genetic integrity of Rhizoctonia spp. In: SNEH, B.; J ABAJ I-HARE, S.; NEATE, S. \& DIJ ST, G., eds. Rhizoctonia species: taxonomy, molecular biology, ecol ogy, pathol ogy, and disease control. Dordrecht, Kluwer Academic Publishers, 1996. p.13-35.

SNEH, B.; BURPEE, L. \& OGOSHI, A. Identification of Rhizoctonia species. Minnesota, APS Press, 1991. 133p.

STALPERS, J .A.; ANDERSEN, T.F. \& GAMS, W. Two proposals to conserve the names Rhizoctonia and R. solani (Hyphomycetes). Taxon, 47:725-726, 1998.

WILKINSON, K.G.; DIXON, K.W.; SIVASITHAMPARAM, K. \& GHISALBERTI, E.L. Effect of IAA on symbiotic germination of an Australian orchid and its production by orchid-associated bacteria. Plant Soil, 159:291-295, 1994.

ZELMER, C.D.; CUTHBERTSON, L. \& CURRAH, R.S. Fungi associated with terrestrial orchid mycorrhizas, seeds and protocorms. Mycoscience, 37:439-448, 1996.

ZETTLER, L.W. Orchid-fungal symbiosis and its value in conservation. Mcl vainea, 13:40-45, 1997a.

ZETTLER, L.W. Terrestrial orchid conservation by symbiotic seed germination: techniques and perspectives. Sebyana, 18:188-194, 1997b. 
Olinto Liparini Pereira et al .

R. Bras. Ci. Solo, 29:191-197, 2005 